\title{
Symptoms of distress among young Danes during the national lockdown in May 2020
}

\author{
Sofie Have Hoffmann ${ }^{1}\left[\right.$ [ $\cdot$ Veronica Sofie Clara Pisinger ${ }^{1}\left[\right.$ ] $\cdot$ Johanne Aviaja Rosing ${ }^{1} \cdot$ Janne S. Tolstrup $^{1}(\mathbb{C}$
}

Received: 9 April 2021 / Accepted: 29 September 2021 / Published online: 20 October 2021

(c) The Author(s), under exclusive licence to Springer-Verlag GmbH Germany 2021

\begin{abstract}
Objectives of this study were to assess (1) prevalence of worries and symptoms of distress, and (2) perceived change in symptoms of distress by sociodemographic factors and preexisting vulnerabilities, among young Danes under the first COVID-19 related lockdown. Data were derived from online surveys, collected 7th-18th of May 2020. The study population included 11,245 young people (15-20 years of age), of which 1807 had participated in The Danish National Youth Study 2019 (DNYS19). Descriptive statistics and linear regressions analyses, including robust standard errors, were performed. All analyses were based on cross-sectional data, except analyses of preexisting vulnerabilities among responders from DNYS19. Few young people were very worried to get infected with coronavirus. Females reported a higher frequency of symptoms of distress than males. Perceived change in symptoms of distress, did not vary systematically based on age, cohabitation, nor physical health conditions. Individuals working, perceived a lower increase in symptoms of distress, than those studying etc. Females with symptoms of anxiety pre-pandemic, mental health disorders, and in families with economic hardship had a marginal higher perceived increase in symptoms of distress, than females without these difficulties. The tendencies were similar but nonsignificant among males, and for symptoms of depression pre-pandemic. In conclusion, during the lockdown, young females reported a higher frequency of symptoms of distress than males, and individuals with symptoms of anxiety pre-pandemic, mental health disorders, and in families with economic hardship were more likely to perceive the lockdown to be associated with an increase in symptoms of distress, than individuals without these difficulties.
\end{abstract}

Keywords Mental health $\cdot$ COVID-19 $\cdot$ Corona $\cdot$ Pandemic $\cdot$ Lockdown $\cdot$ Young

\section{Introduction}

Since early 2020, the world has seen a rapid spread of COVID-19, which on March 11th was defined as a pandemic by WHO. To prevent the virus from spreading further, a wide range of public life restrictions have been introduced around the globe [1], and on the evening of March 11th, the Danish government declared a national lockdown, which meant closing of schools, public workplaces, daycares and restaurants etc. Social distancing was mandated, legal sanctions were introduced against public gatherings of more than 100 people (and later 10), and people were encouraged to stay at home. The social isolation, uncertainty, and (worries about) direct (e.g. relatives and one's own health) and

Sofie Have Hoffmann

sohh@sdu.dk

1 National Institute of Public Health, University of Southern Denmark, Studiestræde 6, 1455 Copenhagen, Denmark indirect effects (e.g. lifestyle and economic constrains) of the pandemic may induce or increase distress. Most research from previous outbreaks, where quarantine has been mandated, show negative psychological impacts that may results in long-term effects [2].

Young people may be especially vulnerable to the indirect effects of the lockdown, being in a period of life marked by detachment from parents and increasing orientation towards peers [3], a desire for greater autonomy, and exploration of sexual identity, which during the lockdown was heavily constrained. Further, mental health problems are more common among young Danes (especially females), than in the rest of the population [4].

The emergent evidence of mental health consequences under the COVID-19 pandemic/lockdown indicate that being young is associated with increased mental distress [5, 6] and the highest levels of depression and anxiety has been found among the youngest adults [7, 8], and the oldest children [9]. Further, studies have shown that females find the 
pandemic more psychologically challenging than males [5, 7, 9-11], and that mental health disorders, physical illness, and low socio economic position is associated with poor mental health during the pandemic lockdown [9, 11-16]. Yet, most of the existing research has solely assessed mental health during the pandemic among adults aged 18 or above $[6,7,13,14,16,17]$, and most studies have no indication of whether differences in distress existed pre-pandemic/lockdown or were caused by different resilience.

Further, in a situation where the infection rates, public restrictions, and economic security vary significantly internationally, generalizability from across boarders may be limited. Danish studies of the COVID-19 pandemics influence on physical and mental health in the Danish population have shown varying results, and all are conducted among individuals aged 18 years and above [17-19]. However, the associations may be different among young Danes, and identification of vulnerable groups, is essential in the efforts to remedy the effects of the pandemic and target preventive interventions.

Therefore, the objectives of this study were to assess (1) prevalence of worries and symptoms of distress, and (2) perceived change in symptoms of distress by sociodemographic factors and preexisting vulnerabilities, among young Danes under the first COVID-19 related lockdown.

\section{Methods}

\section{Study population}

The cohort of BEHavioral and Emotional REsponse to the pandemic lockdown in young people (BEHERE) consists of two independent online surveys and 20 semi-structured telephone interviews made to assess mental health, social relations and alcohol consumption during the Danish lockdown. In the present study, solely survey data will be applied. Both surveys were conducted between the 7th and the 18th of May 2020. While the national lockdown, initiated the 13th of March 2020, to counteract the spread of COVID-19, was still partly upheld. A total of 11,596 young people participated in the BEHERE cohort.

The first sample of the BEHERE cohort (S1) was recruited in the general population of young Danes through educational institutions, non-governmental organizations (NGOs), and social media ads. All young people between 15 and 20 years of age, were eligible to participate. A total of 9727 participated in S1.

The second sample (S2) was recruited among 7000 responders from The Danish National Youth Study 2019 (DNYS19), a national survey focusing on health, health behaviour and wellbeing among students in secondary education, who had provided their phone number and granted researchers the permission to contact them in relation to future studies. A total of 1869 (30\%) participants also agreed to participate in the BEHERE cohort, making longitudinal comparison possible in S2. Further information about the DNYS19 can be found elsewhere [20]. Participants in S2 were all high school students or had just graduated, whereas S1 covered a broader target population. The two surveys were similar; however, some questions were left out in S2, as the information was obtained in DNYS19.

It was clearly stated that participation in the study was voluntary, and participants gave informed consent that their data could be used for research. Data collection was approved by the local Data Protection Agency at the University of Southern Denmark (Jnr.:11.072).

\section{Measures}

\section{Dependent variables}

To obtain information of young people's worries under the lockdown, participants were asked how worried they were about the following issues: (1) getting infected with coronavirus, (2) infecting others with coronavirus, (3) friends or family getting infected with coronavirus, (4) when a normal everyday life would be returning, and (5) when one could see friends and family again. Respond categories ranged from "Very worried" to "Not worried at all".

To retrieve a comprehensive measure of the frequency of symptoms of distress under the lockdown, responders were asked how often after the lockdown they had felt the following: (1) isolated (2) lonely, (3) sad, (4) irritable/in a bad mood, (5) stressed, (6) nervous, and (7) experienced sleeping problems. Respond categories ranged from "Everyday/ almost every day" to "Seldom or never".

To obtain an aggregated score of the respondents perceived change in symptoms of distress associated with the lockdown a scale was constructed. Responders were asked how often they had experienced the symptoms under the lockdown as compared to prior the lockdown (1) felt lonely, (2) felt sad, (3) felt irritable/in a bad mood, 4) felt stressed, 5) felt nervous, and 6) experienced sleeping problems. Isolation was assumed to occur prior in the causal pathway, and, therefore, not included in the scale. Respond categories included: "A lot more often (than prior the lockdown)" [+2], "More often (than prior the lockdown)" [+1], "Unchanged (compared to prior the lockdown)" [0], "More rare (than prior the lockdown)" $[-1]$, and "A lot more rare (than prior the lockdown)" [-2]. The six symptoms were summed to obtain an aggregate score of the responders perceived change in symptoms ranging from -12 to +12 , where negative values indicated the perception of a decrease in 
symptoms, and positive values denoted the perception of an increase in symptoms associated with the lockdown.

\section{Independent variables}

Sociodemographic factors were self-reported and included gender (male, female, other), age ( $\leq 16,17,18$, and $\geq 19$ years old), cohabitation during the lockdown (lives with both parents, one parent, or alone, with friend or others), and occupation during lockdown (primary school, secondary education (e.g. high school), boarding school or folk high school, tertiary education (e.g. university), work, unemployed, and other), and an indicator of the family's economic hardship (parents had no difficulties with bills past year, difficulties with bills past year).

Preexisting vulnerabilities were assessed by measures of health and wellbeing pre the lockdown. Participants in S1, were asked if they had one or more health conditions, which we categorized into physical health conditions and mental health disorders. Mental health disorders and physical health conditions, respectively, were assessed as an indicator of vulnerability.

Among S2 participants, data were derived from the Patient Health Questionnaire-4 (PHQ-4) included in DNYS19, to assess symptoms of depression and anxiety in 2019. This is a validated screening instrument measuring the core symptoms of anxiety and depression, consisting of two questionnaires: General Anxiety Disorder-2 (GAD-2) and Patient Health Questionnaire-2 (PHQ-2) [21, 22]. Responders were asked in 2019 how often, over the last 2 weeks, they had been bothered by four different problems (two core symptoms of anxiety and depression, respectively). Response options were "not at all" [0], "several days" [1], "more than half the days" [2], and "nearly every day" [3]. Scores were summarized for the GAD-2 and PHQ-2, respectively (ranging from 0 to 6 ), and $\geq 3$ were applied as the cut-off points between the normal range and probable cases of anxiety and depression, respectively.

\section{Statistical analyses}

Statistical analyses and data processing were performed using STATA 16.

First, descriptive statistics were applied to assess the prevalence of worries and the frequency of symptoms of distress under the lockdown among males and females, respectively. Second, the perception of change in frequency of symptoms of distress under the lockdown among young Danish males and females was statistically described.

To assess scale reliability of the aggregated measure of participants' perceived change in symptoms of distress Cronbach alpha was calculated. Further, the trend for each of the symptoms summed in the aggregated scale, were assessed separately to assess if some items showed systematic differences.

Second, to assess if perceived change in symptoms of distress varied by sociodemographic characteristics and preexisting vulnerabilities linear regressions were performed. Model assumptions for linear regression were tested in all applied models, to evaluate how the associations of interest may best be assessed. All analyses were stratified by gender to assess potential gender differences. Too few participants reported other gender than male and female for these to be presented separately, and these observations were, therefore, excluded $(n=52)$.

Potential confounders were identified according to existing literature and included age, cohabitation during the Danish lockdown, and occupation. Further to control for potential difference induced by the sampling of participants, all analyses applying both samples were adjusted for sample. All analyses were based on cross-sectional data, except analyses of preexisting vulnerabilities in S2, as these could be derived from DNSY19.

As not all questions were posed in both surveys, the number of individuals included in the different analyses varies, and the frequency of the different factors was stated in all analyses for transparency. The reference group refers to participants with the noted characteristic (varying in the different analyses, and stated in the tables), 17 years old, living with both parents, secondary education, and from sample 1 . Participants with missing information on either covariates or the outcome were excluded (males, $n$ : 114; females, n: 185), and thus, the study population included 11,245 young people (of which 1807 had participated in DNSY19).

\section{Results}

The study population consisted of 2998 males and 8257 females, with an average age of, respectively, 17.9 and 17.7 years (Table 1). Most of the study population were students at secondary education institutions (males: 73\%, females: 69\%), and lived primarily with both parents (who lived together) during the lockdown (males: $64 \%$, females $60 \%$ ). Participants had rarely physical contact with friends (besides from school hours) during lockdown, and $15 \%$ of the males and $12 \%$ of the females never met with friends. However, almost half of the males (45\%) and one-fifth (19\%) of the females met online every day during the lockdown e.g. to video chat or game together.

As presented in Fig. 1, the percentage of females who were very worried during the lockdown was higher, than the percentage among males for all issues included. Young males (1\%) and females (4\%) were not very worried to get infected with coronavirus. However, they worried that they 
Table 1 Baseline characteristics of young Danish males and females in the BEHERE cohort

\begin{tabular}{|c|c|c|}
\hline & \multicolumn{2}{|l|}{ Total $(n=11,245)$} \\
\hline & Males $(n=2988)$ & Females $(n=8257)$ \\
\hline \multicolumn{3}{|l|}{ Age } \\
\hline$\leq 16$ years & $487(16)$ & $1844(22)$ \\
\hline 17 years & $704(24)$ & $1857(22)$ \\
\hline 18 years & $741(25)$ & $1985(24)$ \\
\hline$\geq 19$ years & $1056(35)$ & $2571(31)$ \\
\hline Mean age & 17.9 & 17.7 \\
\hline \multicolumn{3}{|l|}{ Occupation } \\
\hline Primary school & $146(5)$ & $710(9)$ \\
\hline Secondary education (e.g. high school) & $2183(73)$ & $5719(69)$ \\
\hline Boarding school or folk high school & $347(12)$ & $888(11)$ \\
\hline Tertiary education (e.g. university) & $37(1)$ & $140(2)$ \\
\hline Work & $164(5)$ & $517(6)$ \\
\hline Unemployed & $38(1)$ & $78(1)$ \\
\hline Other & $73(2)$ & $205(2)$ \\
\hline \multicolumn{3}{|l|}{ Lives with... } \\
\hline Lives with both parents (who live together) & $1904(64)$ & $4964(60)$ \\
\hline Lives with mother and father on turns (separated parents) & $824(28)$ & $2310(28)$ \\
\hline Lives alone, with friend or others & $260(9)$ & $983(12)$ \\
\hline \multicolumn{3}{|l|}{ Frequency of physical contact with friends after lockdown } \\
\hline Everyday & $219(8)$ & $402(5)$ \\
\hline More than once a week & $535(19)$ & $1539(19)$ \\
\hline Almost every week & $652(23)$ & $2001(25)$ \\
\hline Seldom & $1039(36)$ & $3164(39)$ \\
\hline Never & $428(15)$ & $964(12)$ \\
\hline \multicolumn{3}{|l|}{ Frequency of online contact with friends after lockdown } \\
\hline Everyday & $1295(45)$ & $1562(19)$ \\
\hline More than once a week & $655(23)$ & $1922(24)$ \\
\hline Almost every week & $371(13)$ & $1790(22)$ \\
\hline Seldom & $371(13)$ & $1990(25)$ \\
\hline Never & $181(6)$ & $807(10)$ \\
\hline
\end{tabular}

Values are frequency (\%) unless otherwise specified would infect others (19\% males and 33\% females were very worried of infecting others), and that friends or family would get infected (21\% males and 37\% females were very worried that friends or family would get infected). Further, young males (29\%) and females (48\%) were very worried when their everyday life would return (e.g. when they could go back to school/work), and they could see friends and family again (males: $26 \%$, females: $42 \%$ ).

Between 18 and $67 \%$, of the males and females experienced symptoms of distress every day/almost every day, or more than once a week, during the lockdown, and females more frequently than males reported experiencing symptoms (Fig. 2). For example, approximately one third of the males (35\%) and half of the females (49\%) felt lonely every day/ almost every day or more than once a week.

The majority of young males and females perceived the lockdown to be associated with an increase in symptoms of distress (Fig. 3). For example, 21\% of the males and $25 \%$ of the females felt lonely a lot more often during the lockdown than prior. For all included aspects of distress (feeling isolated, lonely sad, irritable/in a bad mood, nervous stressed, and having sleeping problems), a higher percentage among females, than among males, perceived the lockdown to be associated with an increase in frequency of the symptoms.

The aggregated scale of perceived change in symptoms of distress, showed relatively high internal consistency, with an alpha coefficient for the six items at 0.78 . Further, the tendency of the separated symptoms of distress (summed in the aggregated scale) did not vary systematically (data not shown). Model assumptions for linear regressions were sufficiently fulfilled, in the assessment of the associations presented in Table 2. However, to account for heterogeneity of variance robust standard 


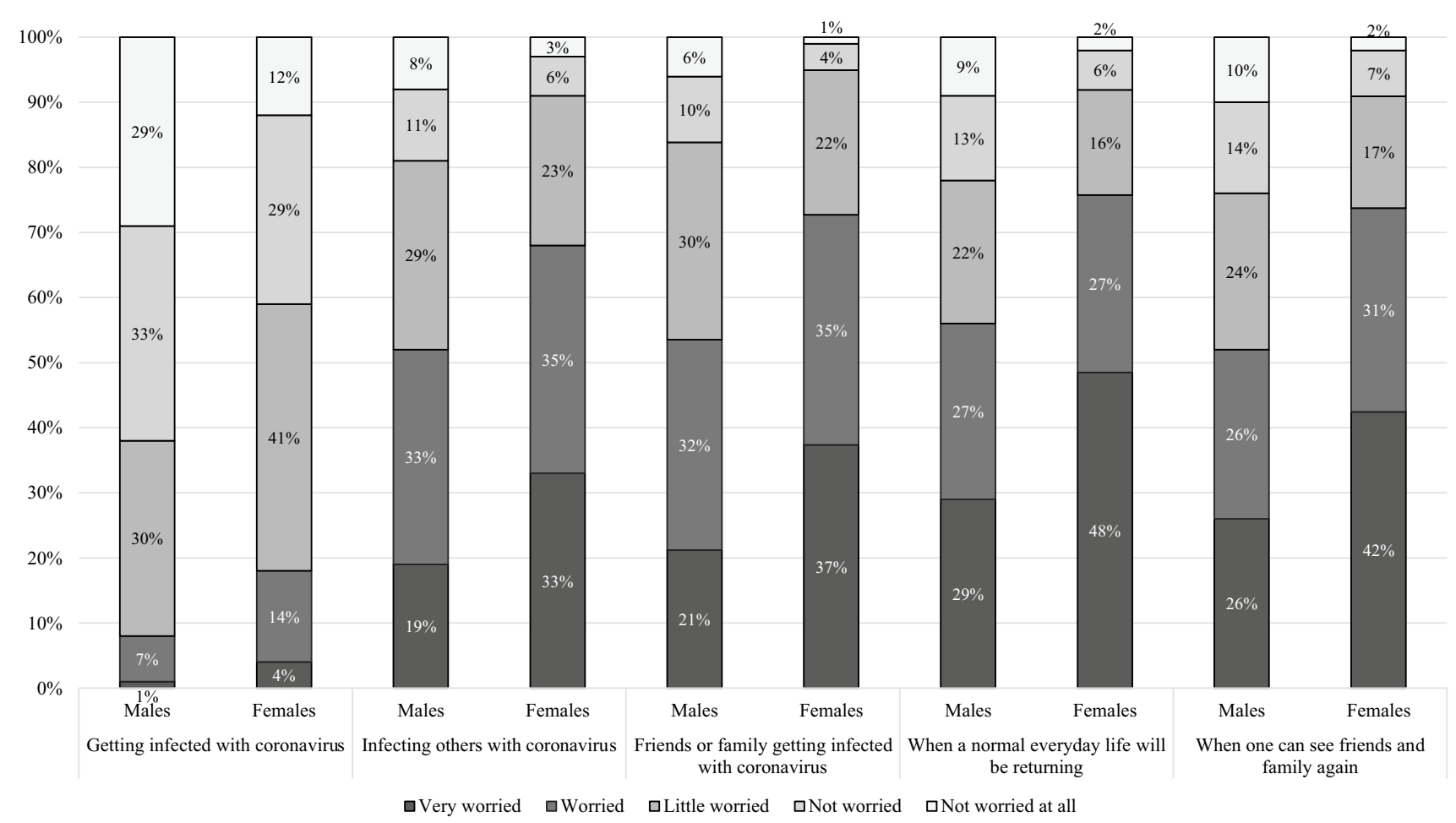

Fig. 1 Worries under the lockdown among Danish males and females in the BEHERE cohort

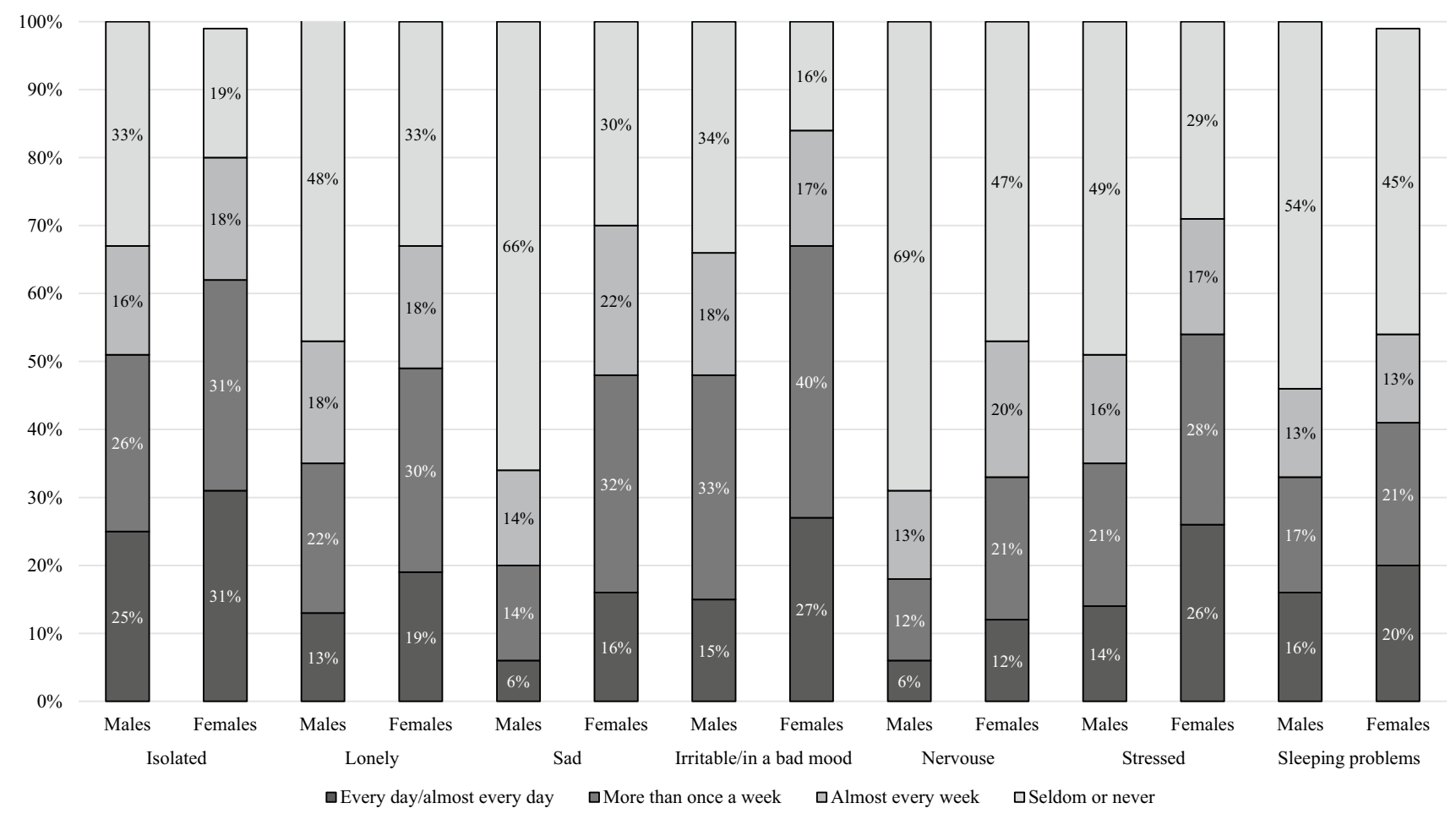

Fig. 2 Frequency of symptoms of distress during the lockdown among Danish males and females in the BEHERE cohort 
errors were estimated. The two approaches showed very similar results with a tendency of a bit more conservative estimates using the robust standard error, and, therefore, only the results of the analyses estimating robust standard errors are presented.

As presented in Table 2, males reported an average perceived increase of 2.02 on the aggregated scale of perceived change in symptoms of distress, while the perceived increase was 3.22 among females.

The perception of change in symptoms of distress did not vary systematically based on responders age or whom they primary lived with. However, participants who worked during the lockdown, had a perception of the lockdown to be associated with a lower increase in symptoms of distress than individuals who studied, were unemployed or had another occupation. Further, males and females reporting that their parents had had difficulties paying their bills within the past year, were more likely to perceive the lockdown to be associated with an increase in symptoms of distress, than males and females who had not experienced this, though results were nonsignificant among males (males: +0.27 $(-0.30 ;+0.83)$, females: $+0.58(+0.27 ;+0.88))$.

In Table 3, the associations between preexisting vulnerabilities and the aggregated scale of perceived change in symptoms of distress are presented. In S1, 25\% of the males and $28 \%$ of the females reported to have a physical health condition, while, respectively, $12 \%$ and $21 \%$ reported to have a mental health disorder. There were $14 \%$ of the males and $29 \%$ of the females in S2 who showed symptoms of anxiety in DNSY 19, and, respectively, 24\% and 33\% showed symptoms of depression. Model assumptions for linear regressions were sufficiently fulfilled, in the assessment of the associations presented in Table 3. However, to account for heterogeneity of variance robust standard errors were estimated. The two approaches showed very similar results with a tendency of a bit more conservative estimates using the robust standard error, and, therefore, only the results of the analyses estimating robust standard errors are presented.

In $\mathrm{S} 1$, young male and females with mental health disorders reported a marginal higher perceived increase in symptoms of distress associated with the lockdown, than young males and females with no mental health disorder(s) ((males: $+0.42(-0.14 ;+0.99)$, females: +0.67 $(+0.43 ;+0.91))$, though the association was nonsignificant among males. Males with physical health conditions reported a marginal higher, but nonsignificant, perceived increase in symptoms of distress compared to males with no physical health conditions $(+0.33(-0.07 ;+0.73))$. Among females no association between physical health

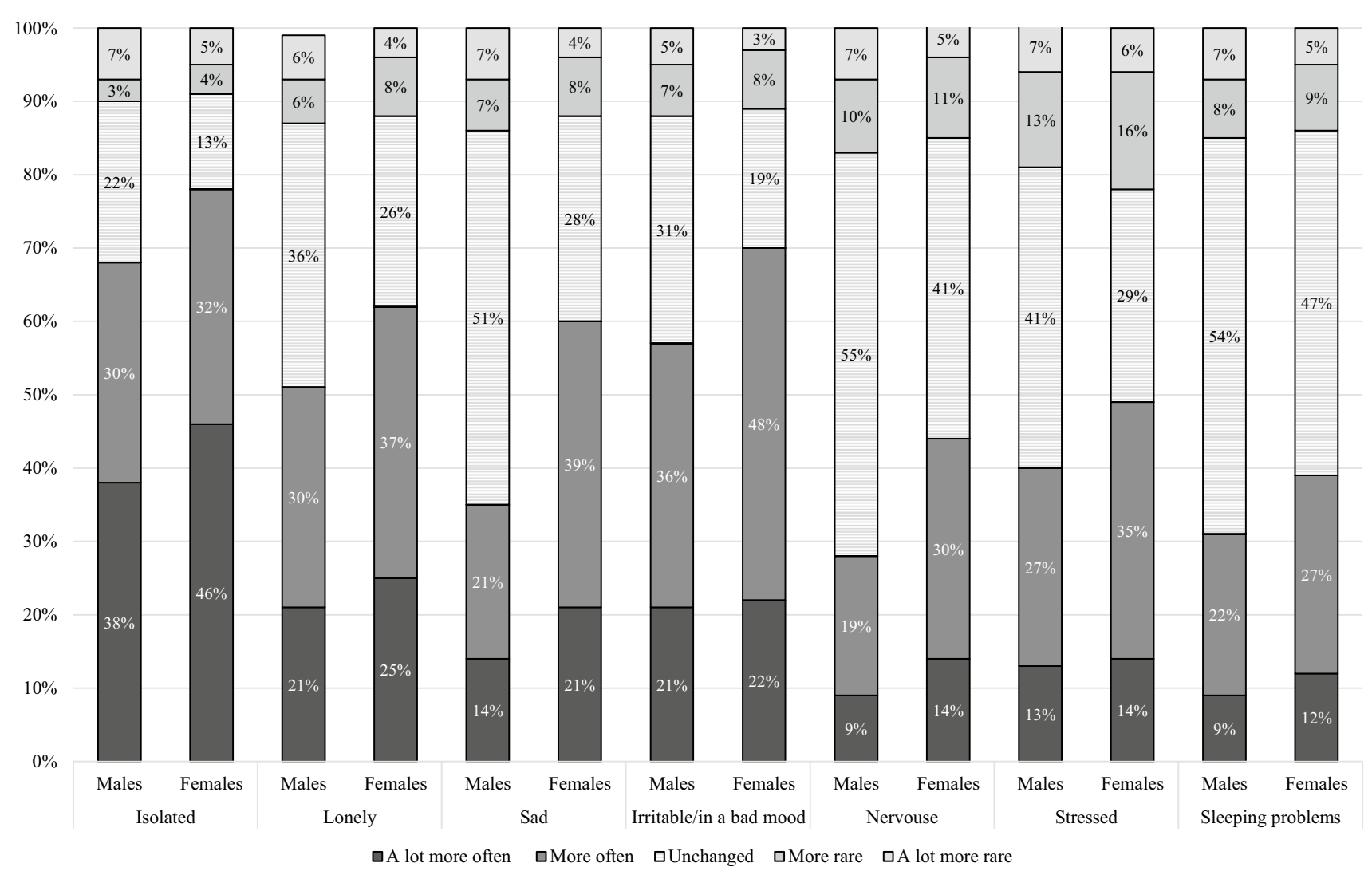

Fig. 3 Perceived change in symptoms of distress associated with the lockdown among Danish males and females in the BEHERE cohort 
Table 2 Mutually adjusted results from linear regression models of perceived change in symptoms of distress associated with the lockdown, among Danish males and females in the BEHERE cohort, by sociodemographic characteristics

\begin{tabular}{|c|c|c|c|c|c|c|c|c|}
\hline & \multicolumn{4}{|c|}{$\begin{array}{l}\text { Males }(n=2998) \\
\text { Perceived change in symptoms of distress associ- } \\
\text { ated with the lockdown, mean: } 2.02\end{array}$} & \multicolumn{4}{|c|}{$\begin{array}{l}\text { Females }(n=8257) \\
\text { Perceived change in symptoms of distress associ- } \\
\text { ated with the lockdown, mean: } 3.22\end{array}$} \\
\hline & Freq. $(\%)$ & Coef & $95 \% \mathrm{CI}$ & $p$ value & Freq. $(\%)$ & Coef & $95 \% \mathrm{CI}$ & $p$ value \\
\hline \multicolumn{9}{|l|}{${ }^{\mathrm{a}} \mathrm{Age}$} \\
\hline$\leq 16$ years old & $487(16)$ & +0.06 & $-0.52 ;+0.65$ & 0.83 & $1844(22)$ & -0.01 & $-0.34 ;+0.32$ & 0.96 \\
\hline 17 years old (ref.) & $704(24)$ & 1.85 & $1.35 ; 2.35$ & - & $1857(22)$ & 3.08 & $2.76 ; 3.40$ & - \\
\hline 18 years old & $741(25)$ & -0.14 & $-0.61 ;+0.32$ & 0.55 & $1985(24)$ & -0.05 & $-0.32 ;+0.22$ & 0.73 \\
\hline$\geq 19$ years old & $1056(35)$ & +0.37 & $-0.08 ;+0.82$ & 0.11 & $2571(31)$ & +0.29 & $+0.02 ;+0.56$ & 0.04 \\
\hline \multicolumn{9}{|l|}{${ }^{a}$ Lives with... } \\
\hline Both parents (ref.) & $1904(64)$ & 1.85 & $1.35 ; 2.35$ & - & $4964(60)$ & 3.08 & $2.76 ; 3.40$ & - \\
\hline Mother or father & $824(28)$ & +0.30 & $-0.04 ;+0.65$ & 0.09 & $2310(28)$ & +0.22 & $+0.02 ;+0.42$ & 0.03 \\
\hline Alone, with friend or others & $260(9)$ & +0.29 & $-0.29 ;+0.88$ & 0.33 & $983(12)$ & -0.01 & $-0.32 ;+0.30$ & 0.95 \\
\hline \multicolumn{9}{|l|}{ accupation } \\
\hline Primary school & $146(5)$ & -0.52 & $-1.35 ;+0.32$ & 0.23 & $710(9)$ & -0.54 & $-0.95 ;-0.12$ & 0.01 \\
\hline Boarding school or folk high school & $2183(73)$ & -0.30 & $-0.93 ;+0.33$ & 0.35 & $5719(69)$ & +0.35 & $-0.01 ;+0.71$ & 0.06 \\
\hline Secondary education (ref.) & $347(12)$ & 1.85 & $1.35 ; 2.35$ & - & $888(11)$ & 3.08 & $2.76 ; 3.40$ & - \\
\hline Tertiary education & $37(1)$ & -0.59 & $-2.22 ;+1.04$ & 0.48 & $140(2)$ & +0.04 & $-0.66 ;+0.75$ & 0.91 \\
\hline Work & $164(5)$ & -1.01 & $-1.67 ;-0.35$ & $>0.01$ & $517(6)$ & -0.55 & $-0.90 ;-0.19$ & $>0.01$ \\
\hline Unemployed & $38(1)$ & +0.01 & $-1.08 ;+1.09$ & 0.99 & $78(1)$ & +0.29 & $-0.50 ;+1.08$ & 0.47 \\
\hline Other & $73(2)$ & -1.23 & $-2.30 ;-0.16$ & 0.02 & $205(2)$ & -0.99 & $-1.58 ;-0.40$ & $>0.01$ \\
\hline \multicolumn{9}{|l|}{ bFamily's economic hardship } \\
\hline No difficulties with bills past year (ref.) & $2370(91)$ & 1.85 & $1.32 ; 2.37$ & - & $6235(87)$ & 3.01 & $2.67 ; 3.34$ & - \\
\hline Difficulties with bills past year & $248(9)$ & +0.27 & $-0.30 ;+0.83$ & 0.36 & $917(13)$ & +0.58 & $+0.27 ;+0.88$ & $>0.01$ \\
\hline
\end{tabular}

${ }^{a}$ Mutual adjusted results and adjusted for sample (not adjusted for family's economic hardship)

${ }^{\mathrm{b}}$ Adjusted for age, living situation, occupation and sample

Reference: the noted characteristic, and 17 years old, living with both parents, secondary education, and sample 1

Table 3 Adjusted results from linear regression models of perceived change in symptoms of distress associated with the lockdown, among Danish males and females in the BEHERE cohort, by pre-pandemic vulnerabilities

\begin{tabular}{|c|c|c|c|c|c|c|c|c|}
\hline & \multicolumn{4}{|l|}{ Males } & \multicolumn{4}{|l|}{ Females } \\
\hline & Freq. $(\%)$ & Coef & $95 \% \mathrm{CI}$ & $p$ value & Freq. (\%) & Coef & $95 \% \mathrm{CI}$ & $p$ value \\
\hline \multicolumn{9}{|l|}{ Sample 1} \\
\hline \multicolumn{9}{|l|}{${ }^{\text {aPhysical health conditions }}$} \\
\hline No physical health condition (ref.) & $1734(75)$ & 1.93 & $1.53 ; 2.33$ & - & $5112(72)$ & 3.16 & $2.93 ; 3.38$ & - \\
\hline Physical health condition & $587(25)$ & +0.33 & $-0.07 ;+0.73$ & 0.10 & 2005 (28) & -0.03 & $-0.25 ;+0.19$ & 0.77 \\
\hline \multicolumn{9}{|l|}{${ }^{\mathrm{a}}$ Mental health disorders } \\
\hline No mental health disorder (ref.) & $2045(88)$ & 1.98 & $1.59 ; 2.36$ & - & $5645(79)$ & 3.04 & $2.82 ; 3.26$ & - \\
\hline Mental health disorder & $276(12)$ & +0.42 & $-0.14 ;+0.99$ & 0.14 & $1472(21)$ & +0.67 & $+0.43 ;+0.91$ & $>0.001$ \\
\hline \multicolumn{9}{|l|}{ Sample 2} \\
\hline \multicolumn{9}{|l|}{${ }^{\text {a}}$ Symptoms of anxiety disorder } \\
\hline Not likely to have anxiety (ref.) & $571(86)$ & 1.74 & $0.67 ; 2.81$ & - & $807(71)$ & 3.11 & $2.35 ; 3.89$ & - \\
\hline Symptoms of anxiety & $90(14)$ & +0.79 & $-0.12 ;+1.70$ & 0.09 & $325(29)$ & +0.53 & $+0.03 ;+1.03$ & 0.04 \\
\hline \multicolumn{9}{|l|}{${ }^{\mathrm{a}}$ Symptoms of depression } \\
\hline Not likely to have depression (ref.) & $503(76)$ & 1.82 & $0.74 ; 2.91$ & - & $759(67)$ & 3.08 & $2.31 ; 3.86$ & - \\
\hline Symptoms of depression & $158(24)$ & +0.22 & $-0.58 ;+1.02$ & 0.59 & $370(33)$ & +0.33 & $-0.17 ;+0.82$ & 0.20 \\
\hline
\end{tabular}

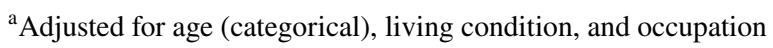

Reference: the noted condition, and 17 years old, living with both parents, and secondary education 
conditions and perception of change in symptoms of distress was observed.

In S2, young males and females who showed symptoms of anxiety in 2019, reported a marginal higher perceived increase in symptoms of distress associated with the lockdown, than their counterparts who did not have symptoms of anxiety in 2019; however, results were nonsignificant among males $(($ males: $+0.79(-0.12 ;+1.70)$, and marginal significant among females $((+0.53(+0.03 ;+1.03))$. A similar tendency was observed for males and females showing symptoms of depression in 2019 , as a marginal higher perceived increase in symptoms of distress was observed, as compared to males and females who did not show symptoms of depression in 2019; however, these results were nonsignificant $(($ males $+0.22(-0.58 ;+1.02)$, females: +0.33 $(-0.17 ;+0.82))$.

\section{Discussion}

With this study, we have shown that a small proportion of young Danish males and females were very worried of getting infected with coronavirus in May 2020, under the first COVID-19 related national lockdown. Further, we have shown that females reported a higher frequency of symptoms of distress under the lockdown than males. These results are in line with studies, among older populations, indicating that females have found the pandemic more psychologically challenging than males [5, 7, 9-11]. However studies among older Danes, has shown conflicting results: one study found no association between sex and worries, emotional distress, nor physical symptom load under the lockdown [19], while another study showed that females were especially negatively affected by the pandemic [18]. To the best of our knowledge, this is the first study to assess young Danes' reactions to the pandemic.

In this study, the perception of change in symptoms of distress, did not vary systematically by age or cohabitation. However, participants who worked during the lockdown, had a perception of a lower increase in symptoms of distress than individuals who studied, were unemployed or had other occupations. This is probably associated with the extent of disruption of everyday life caused by the lockdown. As young individuals who were employed perhaps still could work at the site, and, therefore, see colleagues and maintain a daily routine.

We showed no association between physical health conditions and perceived change in symptoms of distress, as oppose to previous studies among older population, showing an association between preexisting physical health conditions and mental health under the lockdown [16]. This may be related to the broad definition of physical health conditions applied in BEHERE, including non-severe conditions.
Thus, participants may not have been in increased risk of severe outcomes of COVID-19 transmission, and data further showed that there were no association between physical health and level of worries (data not shown). Similar to our results, a Danish longitudinal study among men and women aged 18-72 years, found that physical disease were not associated with illness worry or emotional distress under the lockdown [19].

Our results indicated that females in families with economic hardships were more likely to perceive the lockdown to be associated with an increase in symptoms of distress, as compared to females without these difficulties. The tendencies were similar but nonsignificant among males. Our results are, therefore, in line with other studies that have indicated that low socioeconomic position is associated with increase of mental difficulties after the pandemic among both genders [12], and that the majority of the adult populations are concerned about financial issues associated with COVID-19 [13, 14]. It is interesting that economic hardships repeatedly have been found to be associated with mental difficulties after the pandemic also in our study among young people in the Danish welfare system, where most health care services a free of costs, and the social security system provides financial support to unemployed etc. and relief packages were implemented in response to the pandemic, to reduce financial insecurity [23].

Our results showed that females with symptoms of anxiety pre-pandemic, and mental health disorders perceived the lockdown to be associated with a marginal higher increase in symptoms of distress, than females without these difficulties. The tendencies were similar but nonsignificant among males, and for symptoms of depression pre the pandemic. These results are in line with previous studies indicating that individuals with mental health difficulties have experienced elevated levels of distress in response to the pandemic [11,13-16]. In an Australian study among individuals aged 12-18 years, those with a previous diagnosis of depression and/or anxiety, perceived the pandemic to be associated with a higher worsening of their mental health than those without diagnosis [15]. Yet, most of the existing research has solely assessed mental health during the pandemic among adults aged 18 or above $[6,7,13,14,16,17]$, and most studies have no indication of whether differences in distress existed pre-pandemic/lockdown or were caused by different resilience. Further, the timing of the assessment of mental health during lockdown may be of particular importance. E.g. one study among young adults (aged 18-25) in the UK, found that females' mental health gradually improved from April to September 2020, and then declined again, while males had a relatively stable trajectory across the pandemic [11]. Also, a study among a Chinese population found no differences in the levels of stress, anxiety, and depression from January to March 2020 [24]. In our study data was 
collected, approximately 2 months after the initialization of the national lockdown, while most restrictions initialized the 11th of March were upheld, schools, kindergartens, and other institutions, were gradually reopened from mid-April. Time variations in mental health indicators found among Danes during the lockdown have generally been small, but may vary in different age groups over time [17]. Thus, the long-term effects of the pandemic and the associated restrictions, should be further explored among young Danes, to achieve a better understanding of the mental health and wellbeing in the population. Further, we have not assessed which specific factors that may have induced distress under the national lockdown, nor which factors that may be associated with resilience. Future studies assessing mental health and wellbeing under the COVID-19 lockdown, may benefit from also applying qualitative in-depth analyses to address the mechanisms.

\section{Strengths and limitations}

This study presents the first insight into how the COVID-19 pandemic and the associated restrictions have impacted the mental health of young people living in Denmark. Rapid disseminating of online surveys enabled us to assess mental health, social relations and social patterns among 11,245 young people (15-20 years of age), during the pandemic lockdown in Denmark May 2020. The large study population decreases the impact of non-systematic errors and improves statistical power. Further, participants were recruited across a range of occupations and geographical areas of Denmark, which may increase generalizability of the results. Also, the study incorporates a subsample of measurements of symptoms of anxiety and depression from DNYS19, which provides the opportunity to study pre-pandemic vulnerabilities assessed prior the lockdown.

Some limitations of our study need to be considered. Collecting most of the data at one time point limits the casual inference drawn from the results. Recall bias may occur if respondents could not adequately recall how they felt prior to the national lockdown, they may have over- or underestimated the change in symptoms of distress associated with the lockdown. Further, there may be an underreporting of mental health conditions among responders, as it has previously been found, by comparing self-reports to administrative records, that survey respondents are significantly more likely to under-report mental illnesses compared to other health conditions [25]. This may lead to exposure misclassification, as some may be categorized as not having a mental health disorder, though they actually do, which could lead to an underestimation of the relative difference in perceived change in symptoms of distress, between responders without and with mental health disorders. However, the study incorporates pre-pandemic measures of mental health from DNYS19, showing similar results for reported symptoms of anxiety and depression as for self-reported mental health disorders, and potential exposure misclassification is not expected to have a major impact. Further, one could argue that it is too simplified to dichotomize mental health disorders, as the term covers a wide range of very different diagnoses. However, when we assessed the perceived change in symptoms of distress by mental health disorders the association was similar across disorders (data not shown).

The constructed scale of perceived change in symptoms of distress associated with the lockdown is not a validated measure, and results should be interpreted with caution. However, the scale performed a relatively high internal consistency.

The study population may be subjected to self-selection bias, as young people that identified with the study aims, e.g. they felt strongly impacted by the lockdown, may be more likely to participate, oppositely very distressed individuals may not have the energy to participate. This may hamper representativeness of results. However, among young people invited to BEHERE from DNYS19, there were no systematic differences in in sociodemographic characteristics nor the distribution of symptoms of anxiety and depression in 2019 between responders (constituting S2) and non-responders (data not shown).

Further, distress may manifest differently among males and females, and the composition of the study population (73\% females) may as well impact likelihood of statistically significant results. Nevertheless, our results show an increase in symptoms of distress associated with the lockdown among both genders, and results are in line with existing evidence of gender differences.

\section{Conclusion}

Individuals with symptoms of anxiety pre-pandemic, mental health disorders, and in families with economic hardship were more likely to perceive the lockdown to be associated with an increase in symptoms of distress, than individuals without these difficulties.

Acknowledgements The authors are grateful to the young people participating in the study. Further the authors want to acknowledge Laura Lassen for her contribution in the data collection.

Author contributions All authors designed the study and collected the data. SHH conducted the statistical analyses, formulated, and wrote the first draft of the manuscript. VP and JAR helped in editing and finalizing the manuscript. JST provided significant guidance and helped to edit and write the manuscript.

Funding The Danish Cancer Society. 
Availability of data and materials Not applicable.

Code availability Not applicable.

\section{Declarations}

Conflict of interest Nothing to declare.

Consent to participate It was clearly stated that participation in the study was voluntary, and participants gave consent that their data could be used for research.

\section{References}

1. European Centre for Disease Prevention and Control - An agency of the European Union (2020) COVID-19 pandemic. https://www. ecdc.europa.eu/en/covid-19-pandemic. Accessed 17 Sept 2020

2. Brooks SK, Webster RK, Smith LE, Woodland L, Wessely S, Greenberg N et al (2020) The psychological impact of quarantine and how to reduce it: rapid review of the evidence. The Lancet (British edition) 395(10227):912-920

3. Patton GC, Sawyer SM, Santelli JS, Ross DA, Afifi R, Allen NB et al (2016) Our future: a Lancet commission on adolescent health and wellbeing. The Lancet (British edition) 387(10036):2423-2478

4. Jensen HAR, Davidsen M, Ekholm O, Christensen AI (2018) Danskernes sundhed - Den Nationale Sundhedsprofil 2017: Sundhedsstyrelsen

5. Pierce M, Hope H, Ford T, Hatch S, Hotopf M, John A et al (2020) Mental health before and during the COVID-19 pandemic: a longitudinal probability sample survey of the UK population. TLancet Psychiatry 7(10):883-892

6. Daly M, Sutin A, Robinson E (2020) Longitudinal changes in mental health and the COVID-19 pandemic: evidence from the UK Household Longitudinal Study. Psychol Med: 1-10. https:// doi.org/10.1017/S0033291720004432

7. Fancourt D, Bu F, Mak HW, Steptoe A (2020) Covid-19 social study results-release 24. Department of Behavioural Science \& Health UCL

8. Ribeiro F, Schröder VE, Krüger R, Leist AK (2021) The evolution and social determinants of mental health during the first wave of the COVID-19 outbreak in Luxembourg. Psychiatry Res 303:114090

9. Chen F, Zheng D, Liu J, Gong Y, Guan Z, Lou D (2020) Depression and anxiety among adolescents during COVID-19: a crosssectional study. Brain Behav Immun 88:36-38

10. Liu Y, Yue S, Hu X, Zhu J, Wu Z, Wang J et al (2021) Associations between feelings/behaviors during COVID-19 pandemic lockdown and depression/anxiety after lockdown in a sample of Chinese children and adolescents. J Affect Disord 284:98-103

11. Stroud I, Gutman LM (2021) Longitudinal changes in the mental health of UK young male and female adults during the COVID-19 pandemic. Psychiatry Res 303:114074
12. Chi X, Becker B, Yu Q, Willeit P, Jiao C, Huang L et al (2020) Prevalence and psychosocial correlates of mental health outcomes among Chinese college students during the coronavirus disease (COVID-19) Pandemic. Front Psychiatry 11:803

13. Van Rheenen TE, Meyer D, Neill E, Phillipou A, Tan EJ, Toh WL et al (2020) Mental health status of individuals with a mood-disorder during the COVID-19 pandemic in Australia: initial results from the COLLATE project. J Affect Disord 275:69-77

14. Newby JM, O'Moore K, Tang S, Christensen H, Faasse K (2020) Acute mental health responses during the COVID-19 pandemic in Australia. PLoS ONE 15(7):e0236562

15. Li S, Beames J, Newby J, Maston K, Christensen H, Werner-Seidler A (2021) The impact of COVID-19 on the lives and mental health of Australian adolescents. Eur Child Adolesc Psychiatry. https://doi.org/10.1007/s00787-021-01790-x

16. Iob E, Frank P, Steptoe A, Fancourt D (2020) Levels of Severity of Depressive Symptoms Among At-Risk Groups in the UK During the COVID-19 Pandemic. JAMA Netw Open 3(10):e2026064-e

17. Clotworthy A, Dissing AS, Nguyen T-L, Jensen AK, Andersen TO, Bilsteen JF, et al (2020) 'Standing together-at a distance': documenting changes in mental-health indicators in Denmark during the COVID-19 pandemic. Scandi J Public Health 49:1403494820956445

18. Sønderskov KM, Dinesen PT, Santini ZI, Østergaard SD (2020) The depressive state of Denmark during the COVID-19 pandemic. Acta Neuropsychiatr 32(4):226-228

19. Petersen MW, Dantoft TM, Jensen JS, Pedersen HF, Frostholm L, Benros ME et al (2021) The impact of the Covid-19 pandemic on mental and physical health in Denmark - a longitudinal population-based study before and during the first wave. BMC Public Health 21(1): 1418

20. Pisinger VSC, Thorsted A, Jezek AH, Jørgensen A, Christensen AI, Tolstrup JS, Thygesen LC (2021) The Danish National youth study 2019: study design and participant characteristics. Scand J Public Health:1403494821993724. https://doi.org/10.1177/14034 94821993724

21. Kroenke K, Spitzer RL, Williams JB, Löwe B (2009) An ultrabrief screening scale for anxiety and depression: the PHQ-4. Psychosomatics 50(6):613-621

22. Kroenke K, Spitzer RL, Williams JB, Löwe B (2010) The patient health questionnaire somatic, anxiety, and depressive symptom scales: a systematic review. Gen Hosp Psychiatry 32(4):345-359

23. The Danish Health Authority. High risk groups [in Danish]. https://www.sst.dk/da/corona/Saerlige-risikogrupper. Accessed 17 Nov 2020

24. Wang C, Pan R, Wan X, Tan Y, Xu L, McIntyre RS et al (2020) A longitudinal study on the mental health of general population during the COVID-19 epidemic in China. Brain Behav Immun $87: 40-48$

25. Bharadwaj P, Pai MM, Suziedelyte A (2017) Mental health stigma. Econ Lett 159:57-60 